\section{European Communities Confederation of Clinical Chemistry}

H. Baum

Institut für Laboratoriumsmedizin, Mikrobiologie und Blutdepot, Regionale Kliniken Holding RKH GmbH, Ludwigsburg, Deutschland

\section{Synonym(e) EC4}

Beschreibung Die EC4 wurde von den nationalen Fachgesellschaften der Mitgliedsstaaten der Europäischen Union gegründet. Ein Hauptziel ist, die Fort- und Weiterbildung für alle im Fach Klinische Chemie und Laboratoriumsmedizin tätigen akademischen Professionals europaweit vergleichbar zu gestalten und ein europäisches Register aufzubauen. $\mathrm{Zu}$ sätzlich strebt EC4 eine gegenseitige Anerkennung der Weiterbildung innerhalb der Europäischen Union an. Diese Aufgabe nimmt dabei die „Registration Commission“ (EC4-RC) wahr. Nach der Verschmelzung von EC4 mit der $>$ Forum of the European Societies of Clinical Chemistry and Laboratory Medicine zur $>$ European Federation of Clinical Chemistry and Laboratory Medicine blieb die EC4-RC als selbständige Organisation bestehen.

\section{Literatur}

www.ec-4.org 